\title{
Pedicled lateral nasal wall flap for the reconstruction of the nasal septum perforation. A radio-anatomical study*
}

\author{
Isam Alobid', Eric Mason², C. Arturo Solares², Daniel Prevedello33, Joaquim \\ Enseñat ${ }^{4}$, Matteo De Notaris ${ }^{5,6}$, Alberto Prats-Galino ${ }^{5}$, Manuel Bernal- \\ Sprekelsen ${ }^{1}$, Ricardo Carrau ${ }^{7}$
}

\author{
Rhinology 53: 235-241, 2015 \\ DOl:10.4193/Rhino 14.042 \\ *Received for publication: \\ February 10, 2014 \\ Accepted: February 11, 2015
}

\author{
1 Rhinology and Skull Base Unit, Department of Otorhinolaryngology, Hospital Clinic, Universitat de Barcelona, Spain \\ ${ }^{2}$ Center for Cranial Base Surgery, Department of Otolaryngology, Georgia Regents University, Augusta, GA, USA \\ ${ }^{3}$ Department of Neurosurgery, The Ohio State University Medical Center, Columbus, OH, USA \\ ${ }^{4}$ Department of Neurosurgery, Hospital Clinic, Universitat de Barcelona, Spain \\ ${ }^{5}$ Laboratory of Surgical Neuroanatomy (LSNA), Hospital Clinic, Universitat de Barcelona, Spain \\ ${ }^{6}$ Department of Neuroscience, Division of Neurosurgery, “G. Rummo” Hospital, Benevento, Italy \\ ${ }^{7}$ Comprehensive Skull Base Surgery Centre, Department of Otorhinolaryngology, The Ohio State University Medical Center, Colum- \\ bus, OH, USA
}

\begin{abstract}
Introduction: The aim of this radio-anatomical study is to ensure that the potential donor area of the pedicled lateral nasal wall flap (PLNW) is adequate to reconstruct nasal perforation.

Material and methods: Analysis was conducted on 40 de-identified CT angiographies. The area and length of the PLNW, the septum, and the nasal floor were measured. In a cadaver study, 20 hemi-cranial sagittal sections were also analyzed. The anteriorposterior length of the PLNW flap and the distance between the sphenopalatine foramen and piriform aperture were measured. A clinical study with endoscopic closure of a large perforation was conducted in three patients.

Results: The CT angiographies demonstrated an average PLNW area of $10.80 \pm 1.13 \mathrm{~cm}^{2}$ and a nasal floor area of $3.78 \pm 0.58 \mathrm{~cm}^{2}$. The septal area $\left(22.54 \pm 21.32 \mathrm{~cm}^{2}\right)$ was significantly larger than the total PLNW flap area $\left(14.59 \pm 1.21 \mathrm{~cm}^{2}\right)$. The average length of the flap was $5.58 \pm 0.39 \mathrm{~cm}$, while the septum was $6.66 \pm 0.42 \mathrm{~cm}$; therefore the PLNW flap is insufficient to reconstruct the entire septum. The cadaver study showed that the length of the PLNW flap was $5.28 \pm 0.40 \mathrm{~cm}$. These results demonstrate that measurements obtained from CT scans are reliable data and similar to those found in the radiological study. Complete closure was achieved in all three patients.
\end{abstract}

Conclusion: The PLNW flap does not render enough tissue to reconstruct a total septal perforation; however, up to $84 \%$ of the septum could be repaired with a PLNW. The potential donor area obtained by CT scan and clinical practice support the approachability of PLNW to repair large septal perforation.

Key words: septal closure, pedicle lateral nasal wall flap, septal perforation, septal repair, pedicled nasoseptal flap

\section{Introduction}

Expanded endonasal approaches (EEA) for the treatment of skull base tumours are rapidly evolving. Good exposure, a complete resection, and the subsequent reconstruction of the resultant defect are paramount requirements for an optimum outcome ${ }^{(1-}$ ${ }^{3)}$. In some cases partial or complete septal resection needs to be carried out to achieve an adequate approach or to reconstruct the skull base defect. Subsequently, septal perforation and aesthetic changes may occur in some cases ${ }^{(4)}$.

Other causes of septal perforations include trauma, surgery, inflammatory or infections, neoplasms, or abuse of inhaled 
substances. Nasal septal perforations may be stratified based on location (anterior and posterior) or by size as suggested by Neumann ${ }^{(5)}$ : Small: diameter $\leq 0.5 \mathrm{~cm}$; Medium: diameter between $0.5-2 \mathrm{~cm}$ and Large: diameter $>2 \mathrm{~cm}$. Anterior perforations may cause a sensation of nasal obstruction, crusting, intermittent episodes of epistaxis, malodorous discharge, or result in a whistling sound upon nasal breathing. Perforations of the posterior septum are typically asymptomatic and, as such, rarely require treatment. However, intranasal crusting may be problematic for the patient, especially if the edges of the perforation are not well healed ${ }^{(6)}$.

Non-surgical treatment with frequent lavage with saline irrigations, application of emollients, and antibiotic treatment for infection may ameliorate nasal symptoms. In individuals who remain symptomatic despite the aforementioned treatments, surgical management may be of benefit. Silicone button prosthesis may also relieve these symptoms ${ }^{(7)}$.

The surgical indications and the choice of the ideal reconstruction technique are influenced by the quality of remnants of the septal mucosa that will be used in the repair. In addition, size and location of the perforation are crucial factors in determining the required procedure and type of tissue as well as predicting the success of repair ${ }^{(8)}$. Numerous local endonasal mucosal flaps have been described and the variety of available techniques is evidence enough that no single approach is widely recognized as being reliable to close all perforations. Advancement flaps are currently accepted as standard techniques to close small-medium sized perforations and the flap from the inferior turbinate is advocated for caudal septal perforations ${ }^{(8-10)}$. Open rhinoplasty approaches, the use of temporal parietal-fascia, or facial artery musculomucosal flaps have been also described ${ }^{(11-13)}$.

Recently, a novel technique based on the use of pedicle lateral nasal wall flaps (PLNW), both anterior (APLNW) or posterior (PPLNW), has proven to be a reliable and versatile reconstructive option for extensive defects of the skull base ${ }^{(14,15)}$. A PLNW flap may serve as an alternative procedure to reconstruct large nasal septum defects. However, it is extremely important to be familiar with its vascular anatomy to avoid mucosal damage and flap necrosis. The lateral nasal wall receives blood supply from a multiple anterior, posterior, and superior arterial branches, although its main arterial trunk arises posteriorly from the posterior lateral nasal artery (a branch of the sphenopalatine artery) (Figure 1). It enters the inferior turbinate on the superior aspect of its lateral attachment between 1.0 and $1.5 \mathrm{~cm}$ from its posterior tip. Then, the artery enters a bony canal and bifurcates into two branches. One branch remains high and lateral, while the other runs in a lower and more medial position ${ }^{(16)}$. Moreover, the lateral alar artery (a branch of the facial artery) and the anterior ethmoidal artery supply the anterior and superior areas of the anterior PLNW flap. These blood supplies may allow an anteriorly or pos-

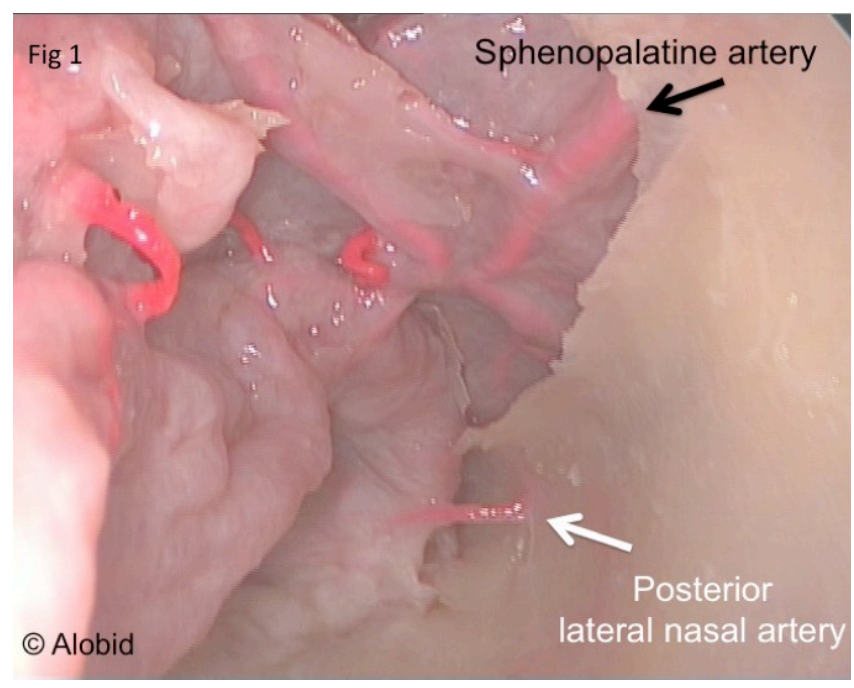

Figure 1. Blood supply of the left lateral nasal wall flap (injected fresh cadaver).

teriorly PLNW flap design to close septal perforations.

To our knowledge, the literature has no data regarding the potential donor site and dimensions of the PLNW flaps and how they might match large septal defects. This radio-anatomical and clinical study was developed to allow optimization of the design of PLNW flaps and to ensure that, when harvesting the flap, its area and length may be adequate to reconstruct large septal defects.

\section{Materials and methods}

\section{Radiological study}

2D projection

Analysis of computed tomographic angiographies (CTA) was conducted on the open source DICOM viewer Osirix (Pixmeo, Switzerland), which has the ability to compute reliable surface areas and distances between landmarks. A random assignment of 20 males and 20 females' CTA was chosen from a de-identified database of CT samples approved by the internal review board. It is a very broad group. All patients were over 18 years of age with no skull base or sinonasal pathology, who underwent CT angiograms for either trauma or intracranial pathology (i.e. aneurysms).

Reliable points were chosen for the flap analysis. We used the sphenopalatine foramen (SPF) as the point of reference to calculate the measurements. The first step was to find the SPF in the saggital plane. This landmark demarcated the most posterior and superior portion of the flap and provided $X-Y$ coordinates that could be extrapolated out of the plane of the SPF and onto the more medial plane of the lateral nasal wall.

The second point was chosen at the floor of the nasal cavity 


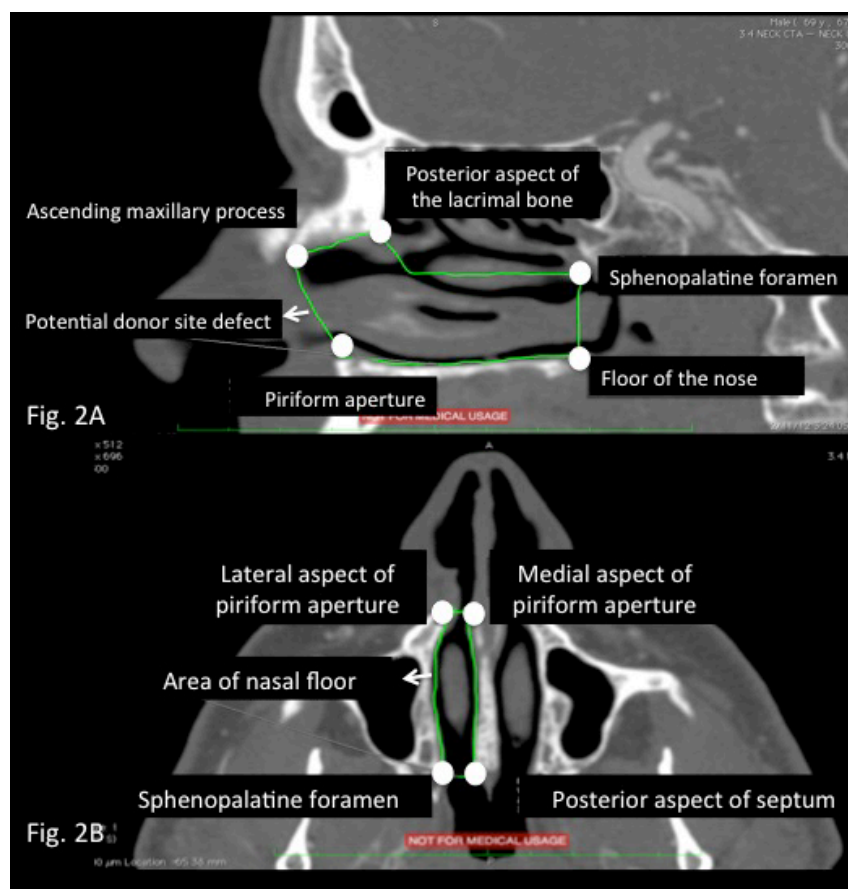

Figure 2. A). Computed tomographic analysis of the lateral nasal wall by Osirix. The first step was to find the sphenopalatine foramen. The second point was chosen at the floor of the nasal cavity inferior to the first point at the same $X$ coordinate. Third point corresponded to the anteriorinferior limit of the flap. The forth and fifth points were chosen over the anterior border of the ascending maxillary process and the posterior aspect of the lacrimal bone, respectively. B) Computed tomographic analysis of the nasal floor. The first point was to find the sphenopalatine foramen. The second point was the posterior aspect of the nasal septum. The forth and fifth points was chosen over the piriform aperture.

inferior to the first point at the same $\mathrm{X}$ coordinate. Moving anteriorly, a third point corresponded to the anterior-inferior limit of the flap at the level of the piriform aperture. The forth and fifth points were chosen at the superior limit of the flap incisions over the anterior border of the ascending maxillary process and the posterior aspect of the lacrimal bone, respectively. With these five points a tracing tool interconnected all, rendering the dimensions of the flap and allowing the calculation of total surface area $\left(\mathrm{cm}^{2}\right)$ (Figure $2 \mathrm{~A}$ ). For additional analysis, the total anterior-posterior distance of the flap, the width of the nasal floor from septum to lateral wall $(\mathrm{cm})$, as well as the total floor area $\left(\mathrm{cm}^{2}\right)$ (Figure 2B) were measured.

For comparison, the total surface area $\left(\mathrm{cm}^{2}\right)$ of the nasal septum was also measured. This required choosing a $\mathrm{CT}$ image that included the entire septum, from nasal floor to the floor of the anterior cranial fossa. The septum was traced with the same tool used for the flap, and the total area and anterior-posterior distance were calculated. This set of measurements represented the potential dimensions of the PLNW flap to close a subtotal septal perforation.
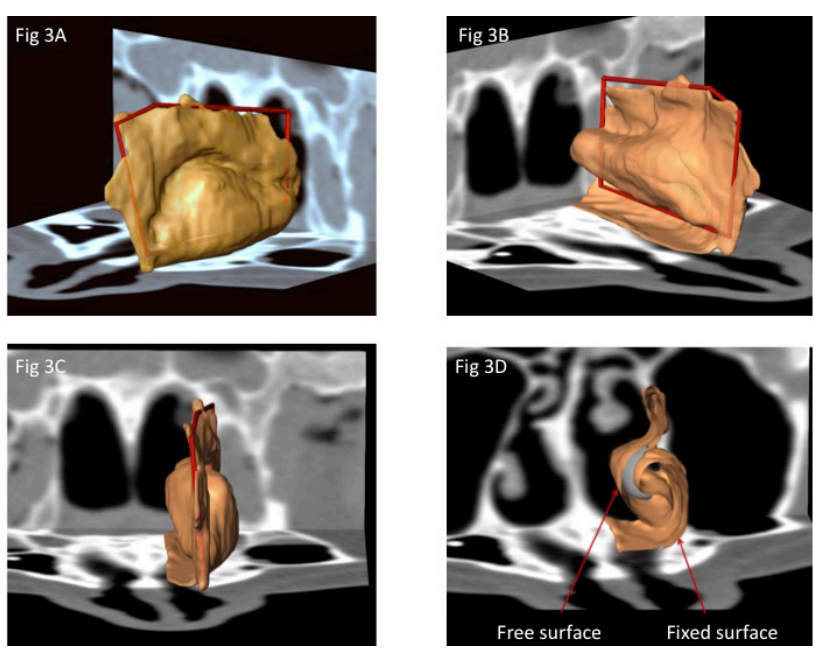

Figure 3. 3D Computed tomographic analysis. A) lateral view, B) medial view, C) frontal view, and D) 3D measurement of potential donor site.

\section{$3 D$ projection}

Fresh injected cadaver was used to compare 3D surface with 2D projection of the PLNW flap. The specimen underwent a preand post-dissection CT scan with a multislice helical acquisition protocol (slice thickness: $0.6 \mathrm{~mm}$; gantry angle $0^{\circ}$ ). The head was positioned in the scanner (Siemens SOMATOM Sensation 64 to obtain a projection perpendicular to the palate. The images achieved were subsequently stored into a PACS (Picture Archiving and Communication System). Each CT image set was stored to create a database of Digital Imaging and Communications in Medicine (DICOM) files. This database was loaded into a Virtual Reality System, known as Dextroscope (Dextroscope; Volume Interactions Pte. Ltd., Singapore) to build up a tridimensional model of the head. The flap was finally removed and the total area of the dissected flap was measured (Figure 3).

\section{Clinical study}

A clinical study was developed to ensure the approachability of this flap to close large perforations. Three patients with symptomatic large septal perforation were included. The septal repair was done endoscopically in two stages. First, the authors closed the perforation using a PLNW flap and 3 months later the pedicle was taken down under general anesthesia. The PLNW flap was designed according to the size and shape of the defect. The floor and lateral nasal wall were infiltrated with a solution of bupivacaine $(0.25 \%)$ containing epinephrine (1:100.000). Two parallel incisions of nasal mucosa were made following frontal process of the maxilla. One incision followed the maxillary line (corresponded intranasally to the junction of the uncinate and maxilla) while a parallel incision followed a line at the piriform aperture reaching the anterior nasal spine. The third anteriorposterior incision was made in the floor to the anterior nasal spine. After removing the turbinal bone, the elevation of the 


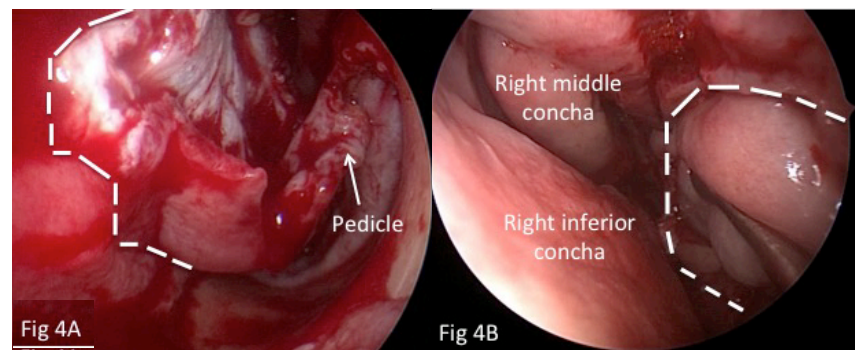

Figure 4. A) Intraoperative view of pedicle lateral nasal wall flap in the left nasal cavity. B) Endoscopica transnasal view of the left flap from right nasal cavity.

mucoperiosteum proceeded in an anterior to posterior direction while the neurovascular pedicle was preserved. The perforation edges were rimmed to obtain fresh margins and the flap was sutured with absorbable suture to the surrounding tissue (Figures $4 A, B)$. Silastic sheets were inserted to support the flap and to prevent adhesions. The floor and the contralateral side of the flap were left bare for closure by secondary intention. Patients were advised to use saline douches to minimize crust formation. Division of the pedicle and suture of the posterior margin of the flap, under general anesthesia, were done 3 months postoperatively.

\section{Cadaver study}

Ten injected fresh cadaver specimens were enrolled in this anatomical study ( 5 females and 5 males). A total of 20 hemi-cranial sagittal sections were analysed. The anterior-posterior length of the PLNW flap as well as the distance between the sphenopalatine foramen and piriform aperture were measured (Figure 5).

\section{Statistical analysis}

Statistics were performed with the SPSS 20.0 (SPSS, Chicago, IL, USA) software. Since our data using Bonferroni correction did not assume normal distribution, a non-parametric test (Wilcoxon test) was used. After completion of measurements, the averages, and standard deviations were calculated with a $95 \%$ confidence interval. A p-value less than 0.05 was considered significant.

\section{Results}

Radiological study

2D projection

Table 1 summarizes all radiological anatomy measurements of 40 CTAs related to the potential donor site and nasal septum, correlating them to specific illustrations. Since there were no significant differences in length, width, and area flap or septum area between males and females, the total measurements were analysed (Table 1). The CT scan study demonstrated an average lateral flap of $10.80 \pm 1.13 \mathrm{~cm}^{2}$ and nasal floor of $3.78 \pm 0.58 \mathrm{~cm}^{2}$.

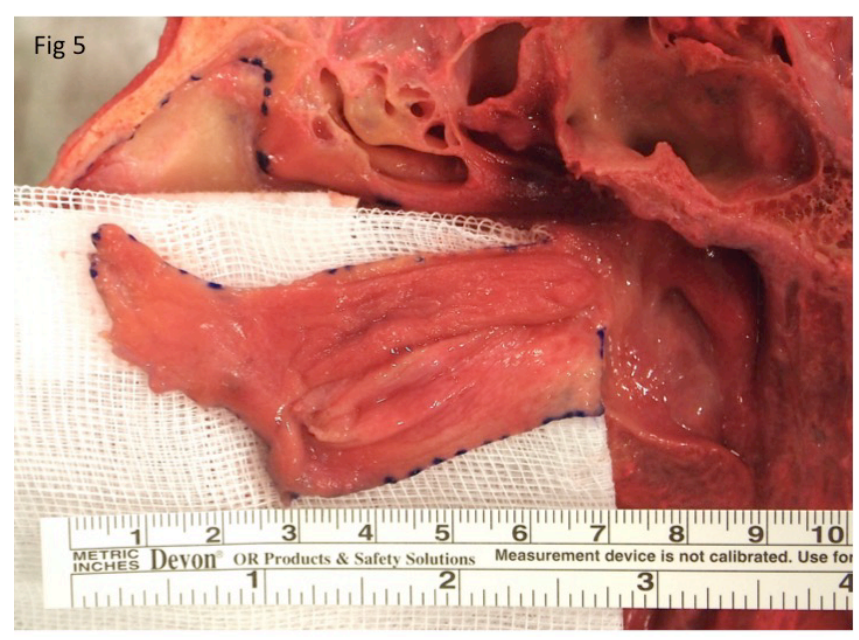

Figure 5. Fresh cadaver dissection of the pedicle lateral nasal wall flap (right side).

On the other hand, the septal area $\left(22.54 \pm 21.32 \mathrm{~cm}^{2}, \mathrm{p}<0.05\right)$ was significantly larger than the total PLNW flap area (14.59 \pm $\left.1.211 \mathrm{~cm}^{2}\right)$. The average length of the flap was $5.58 \pm 0.39 \mathrm{~cm}$ while the septum was $6.66 \pm 0.42 \mathrm{~cm}$. Subsequently, the PLNW flap is insufficient to reconstruct the entire anterior-posterior aspect of the septum.

To measure the optimum size and location of the septal perforation that might be closed by PLNW flap without damaging the pedicle, we calculated the percentage of the anterior and posterior PLNW flaps $(5.58 \mathrm{~cm})$ that could cover the septum $(6.66 \mathrm{~cm})$. The measurements showed that the posterior PLNW flap could cover $83.8 \%$ of the posterior aspect of the septum while the anterior PLNW may cover $83.8 \%$ of the anterior aspect, avoiding excessive flap tension that may produce ischemia and reperforation. Arbitrarily, $5 \mathrm{~mm}$ were added to each approach to allow for some flap retraction that may happen during the scarring. It is possible, however, to use two PLNW flaps for septal repair, thus circumventing this problem. Combined anterior and posterior PLNW flaps from both sides $(5.58 \times 2=11,16 \mathrm{~cm})$ could be an alternative approach for symptomatic total septum perforation $(6.66 \mathrm{~cm})$. Under this condition, there was no need to perform a total PLNW dissection and thereby preventing pedicle damage.

\section{3 p projection}

Nasal mucosa covering the lateral wall and floor of the nose was called "fixed" surface while the mucosa of the inferior turbinate was called "free" surface (Figure 3D). The area of the real dissected PLNW flap was $27.1 \mathrm{~cm}^{2}$. The total 3D estimated donor site (free and fixed surfaces) was $31.2 \mathrm{~cm}^{2}$, however, the total 2D was $14.9 \mathrm{~cm}^{2}$ (the area between 5 points and the floor of the nose). This result demonstrates that the 3D estimated surface is larger than the 2D surface. Moreover, the 2D area is smaller than a real 
Table 1. All measurements related to the pedicle lateral nasal wall flap, nasal floor, and nasal septum.

\begin{tabular}{|c|c|c|c|c|c|}
\hline & Sex & Average & Standard Deviation & $\begin{array}{l}\text { 95\% Confidence } \\
\text { Interval Range }\end{array}$ & $\begin{array}{c}95 \% \text { Confidence } \\
\text { Interval }\end{array}$ \\
\hline \multirow{2}{*}{ Lateral Wall Flap Portion $\left(\mathrm{cm}^{2}\right)$} & Male & 11.06 & 1.16 & $10.55-11.57$ & 0.51 \\
\hline & Female & 10.54 & 1.10 & $10.06-11.03$ & 0.48 \\
\hline \multirow{2}{*}{ Nasal Floor Flap Portion $\left(\mathrm{cm}^{2}\right)$} & Male & 3.89 & 0.75 & $3.56-4.23$ & 0.33 \\
\hline & Female & 3.68 & 0.66 & $3.41-3.95$ & 0.27 \\
\hline \multirow{2}{*}{ Total Flap Area $\left(\mathrm{cm}^{2}\right)$} & Male & 14.95 & 1.17 & $14.44-15.46$ & 0.65 \\
\hline & Female & 14.22 & 1.26 & $13.67-14.77$ & 0.55 \\
\hline \multirow{2}{*}{ Length of Flap (cm) } & Male & 5.68 & 0.35 & $5.53-5.83$ & 0.15 \\
\hline & Female & 5.49 & 0.42 & $5.30-5.67$ & 0.18 \\
\hline \multirow{2}{*}{ Width of Nasal Floor (cm) } & Male & 1.34 & 0.18 & $1.26-1.42$ & 0.08 \\
\hline & Female & 1.28 & 0.12 & $1.23-1.34$ & 0.06 \\
\hline \multirow{2}{*}{ Nasal Septum Area $\left(\mathrm{cm}^{2}\right)$} & Male & 22.54 & 2.45 & $19.28-23.81$ & 1.07 \\
\hline & Female & 21.32 & 2.16 & $20.37-22.26$ & 0.95 \\
\hline \multirow{3}{*}{ Length of nasal Septum (cm) } & Male & 6.64 & 0.38 & $6.38-6.69$ & 0.17 \\
\hline & & & & & \\
\hline & Female & 6.68 & 0.45 & $6.49-6.88$ & 0.20 \\
\hline
\end{tabular}

flap (55\%) and consequently, the estimated area measured by a 2D CT scan is quite reliable for surgical planning. Taking into account the distance between the lateral wall and nasal septum and that the flap may shrink by 20 to $40 \%$ over time, we estimate that any perforation up to half the size of the 2D CT scan could be closed by a PLNW flap.

\section{Clinical study}

Three patients ( $55.7 \pm 7.5$ years; 2 males and 1 female) with large septal perforation ( $34.0 \pm 3.0 \mathrm{~mm}$, large diameter) were operated on and complete closure of their defect was achieved. All perforations were located in the area of the anterior-mid septum. The donor site was left open for closure by secondary intention during 3 months. All patients complained of variable degrees of nasal obstruction due to crusts mainly on the flap side.

\section{Cadaver study}

Hemi-cranial sagittal sections of fresh cadavers were analysed (10 males and 10 females). The anterior-posterior length of the PLNW flap was $5.28 \pm 0.40 \mathrm{~cm}$ without significant differences between males $(5.44 \pm 0.37 \mathrm{~cm})$ and females $(5.12 \pm 0.42 \mathrm{~cm})$. These results are similar to those found in the radiological study (male $5.68 \pm 0.35$ and females $5.49 \pm 0.42$ ) demonstrating that measurements obtained from CT scan are reliable data. Moreover, the distance between the sphenopalatine foramen and the piriform aperture was $4.54 \pm 0.48 \mathrm{~cm}$ without significant differences between males $(4.36 \pm 0.43 \mathrm{~cm})$ and females $(4.72$ $\pm 0.50 \mathrm{~cm}$ ). The resulting distances and areas of our study were similar to the anatomical measures in the study of Lang et al. ${ }^{(17)}$. The total lateral wall length as measured by Lang was $5.18 \mathrm{~cm}$ (range from 3.91 to $6.21 \mathrm{~cm} /$ male 4.80 and female $4.70 \mathrm{~cm}$ ).

\section{Discussion}

Several important points are the result from our study: measurements obtained from 2D and 3D CT scan are reliable data and the clinical study support the approachability of the PLNW flap; the flap is not large enough to reconstruct a total septum perforation; however, up to $83 \%$ of the anterior or posterior nasal perforation can be repaired with a PLNW; the combined use of anterior and posterior PLNW flaps yields a tension-free closure and may be an alternative method for extended septal reconstruction in case of symptomatic patients.

The length and area of the flap in our study were similar to anatomical measures from the Lang et al. study ${ }^{(17)}$. Since the PLNW alone cannot repair a total septal perforation that reaches the columella, the authors propose the use of double anterior 
and posterior flaps to rebuild a new septum. In some clinical cases, using the PLNW, we could achieve a partial or near total closure resulting in the resolution of nasal crusting and nasal obstruction.

Endoscopic expanded skull base approaches to the skull base are techniques that have been greatly improved over the past several years ${ }^{(18)}$. The expansion of surgical indications and the subsequent clinical scenarios have propelled a search for alternative flaps and other methods of reconstruction. Although some of the perforations remain unnoticed by the patients, they may lead to impairment of airflow and are accompanied by a wide variety of symptoms such as nasal obstruction, crusting, discharge, dryness, recurrent epistaxis, pain, and whistling ${ }^{(19)}$. Because the goals of reconstruction are to create a new septum and consequently eliminate crusting, ameliorate breath difficulty, and avoid aesthetic changes, the dimensions of the flap must be adequate to cover almost all of the defect.

The current medical literature describes countless techniques for closing septal perforations, which may be a reflection of the complexity of any such surgical undertaking. Complete anatomical closure of symptomatic total septal perforations is still challenging; usually the outcome is only partially successful and further surgery is needed ${ }^{(20)}$. Repair of septal perforation can be performed using either closed or open approaches. The former is less invasive, minimizes healing time due to rapid mucosalization, and does not leave any external scars, but it is a more difficult technique due to the limited exposure of the surgical field ${ }^{(9,11)}$. The difficulty of performing mucosal suturing via an endonasal approach limits the possibility of applying conventional surgical techniques that are used in open approaches, such as pericranium flap, temporal muscle, etc. Patients in whom local advancement flaps from the septum have failed need flaps from other areas. Those who have had extensive previous septal surgery with cartilage removal are no candidates for local advancement flaps.

Historically, pedicled inferior turbinate flaps have been used for the closure of small nasal septal perforations ${ }^{(21,22)}$. Larger perforations are not suitable to be addressed with this flap.

Understanding the potential dimensions of the septal defect and PLNW flaps vascularization aids the surgeon to achieve the best possible outcome. The size and location of nasal perforation should be considered when planning to repair the septum. PLNW flaps have a rich vascular supply based on the branch of the posterolateral nasal artery that, in turn, is a branch of the sphenopalatine artery. Branches of the facial and ethmoidal arteries provide supply for the anterior PLNW.

Anatomical dissections of fresh cadaver specimens demonstrated that the inferior turbinate mucosal flap provides $4.97 \mathrm{~cm}^{2(23)}$.
However, our radiological study demonstrates that additional mucosa extending beyond the lateral nasal wall, including the mucoperiosteum of the inferior lateral wall, inferior meatus, nasal floor, and Agger Nasi can be harvested to increase the surface area of the PLNW up to $14.5 \mathrm{~cm}^{2}$. Consequently, the surface area of these enlarged flaps may be three times greater than the inferior turbinate flap.

The advantages of the PLNW flap are abundant blood supply and easy rotation. Additionally, the flap consists of respiratory tract mucosa, which allows the repaired septum to achieve normal physiology. The major disadvantage of the PLNW is the requirement for a second procedure to release the pedicle. Another issue is that the flap is bulky and may cause partial nasal obstruction. In addition, the donor surface of the flap must heal by secondary intention producing crusting during the healing process ${ }^{(10,24)}$. Previous studies using inferior turbinate demonstrated no signs of atrophic rhinitis or ozaena secondary to the use of turbinate flap ${ }^{(10,23)}$.

This study is the first attempt in the literature to obtain PLNW flap measurements in reference to its potential to repair a septal perforation. Standardization may, however, not be possible because of the anatomical variations.

\section{Study limitations}

Landmarks and areas measured by the CT scan study don't exactly match with endoscopic findings. The middle meatus between the upper aspect of the inferior turbinate and the lower edge of the uncinate process (maxillary sinus fontanelle) has membranous tissue and usually it is difficult to dissect. However, the mucosa of the medial aspect of the inferior meatus (area not calculated due to turbinate collapse and overlapping with lateral mucosa of the inferior meatus) could be the compensatory area of the fontanelle.

Although dimensions of the PLNW flap are suitable to repair septal perforation, there is no evidence that the blood supply is appropriate for the whole flap areas. Further studies, using quantitative assessment of perfusion and vascular feasibility by angiography, are recommended to validate our pilot study.

\section{Conclusions}

Reconstruction of huge or near total septal defects remains a challenging problem. Using pedicled mucoperiosteal flaps allows for maintaining normal nasal physiology. The current study demonstrates that anterior and posterior PLNW flaps are viable alternatives to repair symptomatic large defects, and each one may cover approximately up to three quarters of the nasal septum. Familiarity with nasal blood supply and understanding of the potential dimensions of the septal defect allow for more op- 
tions to correct a difficult problem. This radio-anatomical study demonstrates that CT scan data and clinical practice support the approachability of PLNW to repair large septal perforation.

\section{Acknowledgements}

This study was support partially by the European Rhinologic Society - Clinical Research Grant 2013-in cooperation with the Foundation of Rhinology and Facial Surgery (RHiPla-Stifung Ulm, Germany).

\section{Author contributions}

IA: conception and design; acquisition and interpretation of data; drafting of the manuscript. EM: acquisition of data and critical revision of the manuscript. CAS: analysis and interpretation of data; drafting of the manuscript. DP, MDN, APG, MBS: acquisition of data and critical revision of the manuscript. RC: conception and design; acquisition and interpretation of data; drafting and revision of the manuscript.

\section{Conflict of interest}

No conflict of interest.

\section{References}

1. Alobid I, Enseñat J, Mariño-Sánchez F, et al. Expanded endonasal approach using vascularized septal flap reconstruction for skull base tumors has a negative impact on sinonasal symptoms and quality of life. Am J Rhinol Allergy. 2013; 27: 426-431.

2. Alobid I, Enseñat J, Mariño-Sánchez F, et al. Impairment of olfaction and mucociliary clearance after expanded endonasa approach using vascularized septal flap reconstruction for skull base tumors. Neurosurgery. 2013; 72: 540-546.

3. Georgalas C, Badloe R, van Furth W, Reinartz S, Fokkens WJ. Quality of life in extended endonasal approaches for skull base tumours. Rhinology. 2012; 50: 255-261.

4. Kasemsiri P, Carrau RL, Otto BA, et al. Reconstruction of the pedicled nasoseptal flap donor site with a contralateral reverse rotation flap: Technical modifications and outcomes. Laryngoscope. 2013; 123: 811 815.

5. Neumann A, Morales-Minovi CA, SchultzCoulon HJ. Closure of nasal septum perforations by bridge flaps. Acta Otorrinolaringol Esp. 2011; 62: 31-39.

6. Certal V, Silva H, Santos T, Correia A, Carvalho C. Trans-septal suturing technique in septoplasty: a systematic review and meta-analysis. Rhinology. 2012; 50: 236-245.

7. Døsen LK, Haye R. Silicone button in nasa septal perforation. Long term observations. Rhinology. 2008; 46: 324-327.

8. Pignatari S, Nogueira JF, Stamm AC. Endoscopic "crossover flap" technique for nasal septal perforations. Otolaryngol Head Neck Surg. 2010; 142: 132-134.

9. André RF, Lohuis PJ, Vuyk HD. Nasal septum perforation repair using differently designed, bilateral intranasal flaps, with nonopposing suture lines. J Plast Reconstr Aesthet Surg. 2006; 59: 829-834
10. Friedman M, Ibrahim $H$, Ramakrishnan V. Inferior turbinate flap for repair of nasal septal perforation. Laryngoscope. 2003; 113: 1425-1428.

11. Kridel RW, Appling WD, Wright WK. Septal perforation closure utilizing the external septorhinoplasty approach. Arch Otolaryngol Head Neck Surg. 1986; 112 168-172.

12. Nuñez-Fernández D, Vokurka J, Chrobok V. Bone and temporal fascia graft for the closure of septal perforation. J Laryngol Otol. 1998; 112: 1167-1171.

13. Xie L, Lavigne F, Rahal A, Moubayed SP, Ayad T. Facial artery musculomucosal flap for reconstruction of skull base defects: a cadaveric study. Laryngoscope. 2013; 123: 1854-1861.

14. Hadad G, Rivera-Serrano CM, Bassagaisteguy LH, et al. Anterior pedicle lateral nasal wall flap: a novel technique for the reconstruction of anterior skull base defects. Laryngoscope. 2011; 121: 1606 1610.

15. Rivera-Serrano CM, Bassagaisteguy $L H$, Hadad G, et al. Posterior pedicle lateral nasal wall flap: new reconstructive technique for large defects of the skull base. Am J Rhinol Allergy. 2011; 25: e212-216.

16. Padgham N, Vaughan-Jones R. Cadaver studies of the anatomy of arterial supply to the inferior turbinates. J R Soc Med 1991; 84: 728-730

17. Lang J. Clinical Anatomy of the Nose, Nasal Cavity and Paranasal Sinuses. Ed Thieme, 1989.

18. Kassam AB, Prevedello DM, Carrau RL, et al Endoscopic endonasal skull base surgery: analysis of complications in the authors initial 800 patients. J Neurosurg. 2011; 114: 1544-1568.

19. Teymoortash A, Hoch S, Eivazi B, Werner JA. Experiences with a new surgical tequnique for closure of large perforation of the nasal septum in 55. Am J Rhinol Allergy. 2011; 25: 193-197.

20. Romo T. A graduated approach to the repair of nasal septal perforations. Plast Reconstr Surg 1999; 103: 66-75.

21. Masing H, Gammert C, Jaumann MP: Our concept concerning treatment of septal perforations. Laryngol Rhinol Otol 1980, 59: 50-56.

22. Vuyk HD, Versluis RJ: The inferior turbinate flap for closure of septal perforations. Clin Otolaryngol Allied Sci. 1988; 13: 53-7.

23. Murakami CS, Kriet JD, lerokomos AP. Nasal reconstruction using the inferior turbinate mucosal flap. Arch Facial Plast Surg. 1999; 1:97-100.

24. Yip J, Macdonald KI, Lee J, Witterick IJ, et al. The inferior turbinate flap in skull base reconstruction. J Otolaryngol Head Neck Surg. 2013; 42: 6-11.

25. Streiner DL. The case of the missing data: methods of dealing with dropouts and other research vagaries. Can J Psychiatry. 2002; 47: 68-75.

Isam Alobid

Rhinology and Skull Base Unit

Dept of Otorhinolaryngology

Hospital Clínic

Universitat de Barcelona

c/ Villarroel, 170

Barcelona 08036 Spain

Tel: $+34-932-279872$

Fax: +34-932-275 050

E-mail: isamalobid@gmail.com 